\title{
CAN LOCALIZATION MICROSCOPY BENEFIT FROM APPROXIMATION THEORY?
}

\author{
Hagai Kirshner, Cédric Vonesch, Michael Unser \\ Biomedical Imaging Group, EPFL, Lausanne, Switzerland
}

\begin{abstract}
We introduce a general and computationally efficient approach to 3-D localization microscopy. The main idea is to construct a continuous-domain representation of the PSF by expanding it in a polynomial B-spline basis. This allows us to fit the PSF to the data with sub-pixel accuracy. Since the basis functions are compactly supported, the evaluation of the PSF is computationally efficient. Furthermore, our approach can accommodate for any 3-D PSF design, and it does not require a calibration curve for the axial position. We further introduce a computationally efficient implementation of the least-squares criterion and demonstrate its potential use for fast and accurate reconstruction of super-resolution data.
\end{abstract}

\section{INTRODUCTION}

The field of localization microscopy has a fundamental role in studying the 3-D structure of the living cell. In particular, super-resolution fluorescence microscopy is based on exciting a sparse set of fluorophore markers at each time instant, and on localizing each of them individually [1]. Localization amounts to fitting the acquired images of single fluorophores with the PSF (point spread function) of the microscope.

A possible approach to the fitting task is maximizing the likelihood function of each acquired image. This is a computationally demanding task that requires additional knowledge on the noise sources. It relies on optimization procedures that are in many cases involved in terms of the cost function and in terms of the numerical implementation [2]. A computationally more efficient approach is the least-squares criterion, which relies on the PSF model only. While it is just slightly inferior to maximum-likelihood formulations in terms of localization accuracy (a difference in standard deviation of 2 [nm] was reported in [3]), it lends itself to fast minimization using the Levenberg-Marquardt algorithm.

Further improvement in computational time can be achieved by Gaussian approximation. This, in turn, allows for real-time 2-D super-resolution reconstruction $[4,5,6]$. Such an approximation was successfully applied to 3-D imaging, too $[7,8]$. Instead of using a conventional widefield set-up, imaging was carried out using a modified pattern that better

This work was funded in part by the Swiss National Science Foundation under Grant 200020-12176 and by the Euro BioImaging project encodes the axial position of the particle. The advantage of these methods resides in their simplified parametrization of the 3-D pattern, which encodes the particle's axial position by a single parameter. The astigmatic PSF, for example, is assumed to be a Gaussian function with varying ellipticity parameter, and the double helix pattern can be described by two rotating lobes. Nevertheless, the true PSF does not necessarily correspond to such simplified models, and such specificity prevent them from being used by other PSF designs. Extracting the calibration curve is another limitation of the simplified parametrization approach.

We propose in this work a general method for 3-D particle localization. It can accommodate for any PSF model, and it does not require a calibration curve. We approximate the continuous-spatial 3-D PSF by means of polynomial Bsplines, using a measured or a computed z-stack of fixed fluorophores for doing so. This type of approximation is guaranteed to have the minimum possible approximation error bound for a given sampling step size. Since the building blocks of our 3-D approximation are compactly-supported functions, they allow for a computationally efficient interpolation method. We also introduce a computationally efficient minimization of the least-squares criterion and demonstrate its potential use for fast and accurate reconstruction of superresolution data.

\section{B-SPLINE REPRESENTATION OF 3-D PSFS}

Let $h\left(x_{d}, y_{d} ; x_{p}, y_{p}, z_{p}\right)$ be a 3-D PSF. The particle position is $\left(x_{p}, y_{p}, z_{p}\right)$ and the detector position is $\left(x_{d}, y_{d}\right)$. We discretize $h$ by evaluating it on the $\left(x_{d}, y_{d}, z_{p}\right)$ grid (Figure 2)

$$
h[k, l, m]=h(\underbrace{k \Delta_{x}, l \Delta_{y}}_{\text {detector }} ; \underbrace{0,0, m \Delta_{z}}_{\text {particle }}) .
$$

Here, $\Delta_{x}, \Delta_{y}$ are the pixel dimensions, $\Delta_{z}$ is the axial step size, and $0 \leq k<n_{x}, 0 \leq l<n_{y}, 0 \leq m<n_{z}$. We exploit this 3-D data for approximating $h\left(x_{d}, y_{d} ; x_{p}, y_{p}, z_{p}\right)$ by means of polynomial splines

$$
\begin{aligned}
& \hat{h}\left(x_{d}, y_{d} ; x_{p}, y_{p}, z_{p}\right)=\sum_{k=0}^{n_{x}-1} \sum_{l=0}^{n_{y}-1} \sum_{m=0}^{n_{z}-1} c[k, l, m] . \\
& \cdot \beta^{n}\left(\frac{x_{d}-x_{p}}{\Delta_{x}}-k\right) \beta^{n}\left(\frac{y_{d}-y_{p}}{\Delta_{y}}-l\right) \beta^{n}\left(\frac{z_{p}}{\Delta_{z}}-m\right) .
\end{aligned}
$$


Here, $\beta^{n}$ is a polynomial B-spline of degree $n$, and $c \in$ $\mathbb{R}^{n_{x} \times n_{y} \times n_{z}}$ is uniquely determined by interpolating the sample values of (1). The PSF is a smooth function and the approximation error that is introduced by $\hat{h}$ is guaranteed to converge to zero with decreasing values of the 3-D sampling volume. Assuming $h$ has $n+1$ derivatives in $L_{2}$, the rate of convergence is given by

$$
\|h-\hat{h}\|_{L_{2}}=\mathcal{O}\left(\Delta^{n+1}\right),
$$

where $\Delta=\max \left(\Delta_{x}, \Delta_{y}, \Delta_{z}\right)$ and $n$ is the B-spline degree we use for constructing $\hat{h}$. Evaluating $\beta^{n}$ requires monomials of degree $n$. Their compact support leads to a computationally efficient interpolation procedure for $\hat{h}$; only few representation coefficients and shifted versions of $\beta^{n}$ are required for evaluating $\hat{h}$ at a single point.

The dimensions of the PSF stack impose no limitation on calculating $c$. We take advantage of the B-spline theory and apply a single digital filter to all three directions of the z-stack. The filter is an all-pole filter and is uniquely determined by the B-spline degree [9]. The B-spline generating function $\beta^{n}$ is compactly supported, having the minimum possible support for a given approximation order. This means that for a given approximation order, the values of $\hat{h}$ are determined by the minimum possible number of representation coefficienst $c$. Moreover, the piecewise polynomial structure of $\beta^{n}$ allows for fast interpolation, too.

\section{PSF FITTING}

Our fitting algorithm consists of local maxima identification and of single particle localizations. The threshold for the local maxima is determined by the noise level, which is estimated from the data. We identify local maxima in each frame and make sure that no two local maxima are too close. We fit the neighborhood of every local maximum with our continuous PSF model. Mathematically, we minimize the least-square error between the pixel values $d[i, j]$ and shifted versions of $\hat{h}$

$$
\min _{A, x_{p}, y_{p}, z_{p}} \sum_{k=0}^{n_{x}} \sum_{l=0}^{n_{y}}\left[d[k, l]-A \hat{h}\left(k \Delta_{x}, l \Delta_{y} ; x_{p} . y_{p}, z_{p}\right)\right]^{2} \text {. }
$$

We utilize the Levenberg-Marquardt method and set the initial parameters as follows $\left(\left(k_{0}, l_{0}\right)\right.$ denote the pixel of the local maximum pixel):

- $\left(x_{p}, y_{p}\right)=\left(k_{0} \Delta_{x}, l_{0} \Delta_{y}\right)$ $\left(k_{0}, l_{0}\right)$ are the coordinates of the local maximum with respect to the upper-left corner of its neighborhood.

- $z_{p}=m \Delta_{z}$ where $m=\underset{m}{\arg \max } \frac{\langle d, h[\cdot, \cdot, m]\rangle}{\|d\|\|h[\cdot, \cdot, m]\|}$ is the slice with the maximum correlation with the data

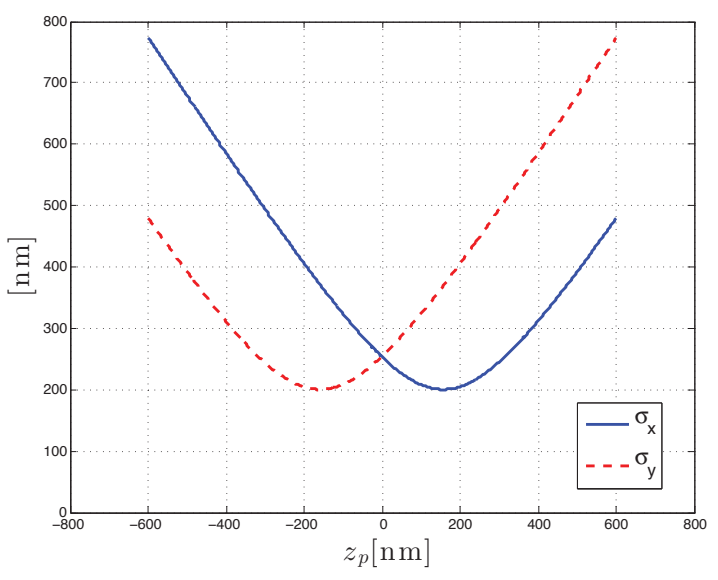

Fig. 1. The astigmatic PSF: Shown here are $\sigma_{x}$ and $\sigma_{y}$ of (7) as a function of the particle depth position $z_{p}$. The acquisition parameters are $\lambda=668[\mathrm{~nm}], \mathrm{NA}=1.4, n_{i}=1.515$. The model constants are $w_{0}=0.21 \lambda / \mathrm{NA}, c=200[\mathrm{~nm}], \alpha=0.79$ and $d=0.2 \lambda n_{i} / \mathrm{NA}^{2}$.

$$
\text { - } A=\frac{d\left[k_{0}, l_{0}\right]}{\hat{h}\left(k_{0} \Delta_{x}, l_{0} \Delta_{y} ; k_{0} \Delta_{x}, l_{0} \Delta_{y}, m \Delta_{z}\right)}
$$

The Levenberg-Marquardt algorithm requires partialderivative values of $\hat{h}$, which can be evaluated by the following relation

$$
\frac{d}{d x} \beta^{n}(x)=\beta^{n-1}\left(x+\frac{1}{2}\right)-\beta^{n-1}\left(x-\frac{1}{2}\right) .
$$

Once a particle is localized, we examine its validity. We do not allow its lateral position to deviate too much from the center of the local maximum. We also exclude fitting results that yield too low SNR values.

\section{RESULTS}

Our algorithm was implemented in Java as an ImageJ plugin. We simulated the astigmatic PSF by [7],

$$
h\left(x_{d}, y_{d} ; x_{p}, y_{p}, z_{p}\right)=A e^{-2\left[\frac{\left(x_{p}-x_{d}\right)^{2}}{\left[\sigma_{x}\left(z_{p}\right)\right]^{2}}+\frac{\left(y_{p}-y_{d}\right)^{2}}{\left[\sigma_{y}\left(z_{p}\right)\right]^{2}}\right]},
$$

where

$$
\sigma_{x}\left(z_{p}\right)=\sigma_{y}\left(-z_{p}\right)=\sigma_{0} \sqrt{1+\frac{\left(\alpha z_{p}-\beta\right)^{2}}{d^{2}}}
$$

The parameter $\sigma_{0}$ describes the Gaussian PSF at focus and we chose its value to be $\sigma_{0}=2 \cdot 0.21 \lambda / \mathrm{NA}$ [10], where $\lambda=668[\mathrm{~nm}], \mathrm{NA}=1.4$. The parameter $\beta$ corresponds to the particle depth value for which $\sigma_{x}$ or $\sigma_{y}$ is minimal, and we chose it to be $\beta=200[\mathrm{~nm}]$ according to Figure 1B of [7]. The scaling constant $\alpha=0.79$ accounts for the refractive index mismatch between the immersion layer $\left(n_{i}=1.515\right)$ and the sample layer $\left(n_{s}=1.35\right)$ [7]. The parameter $d$ is related 


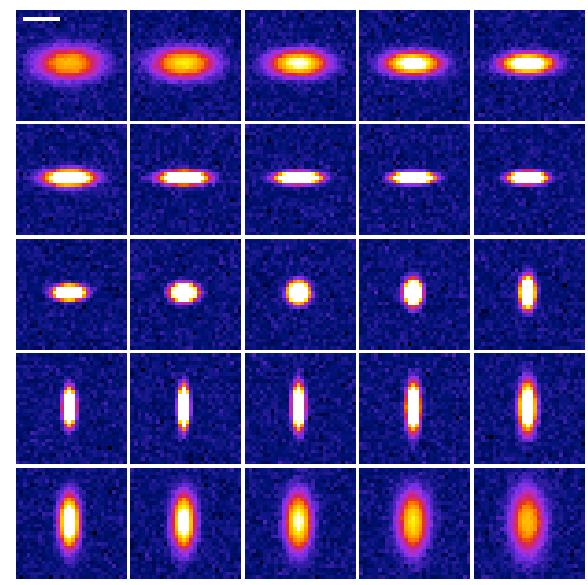

Fig. 2. An astigmatic PSF stack: The model parameters are given in Figure 1, and the size of the stack is $31 \times 31 \times 25$. The pixel size is $\Delta_{x, y}=150[\mathrm{~nm}]$ and the $z_{p}$ step size is $\Delta_{z}=40[\mathrm{~nm}]$. The emission rate of the particle is $2 \cdot 10^{6}[\mathrm{photons} / \mathrm{sec}]$ and the acquisition time is $1[\mathrm{sec}]$. The readout noise has an RMS (Root Mean Square) value of $20[$ electron/pixel]. The upper left pattern corresponds to $z_{p}=-500[\mathrm{~nm}]$ and the scale bar is 10 pixels wide, corresponding to a length of $1500[\mathrm{~nm}]$

to the depth-of-field, and we chose it to be $d=0.5 \lambda n_{i} / \mathrm{NA}^{2}$ according to Figure 1B of [7], too. The functions $\sigma_{x}\left(z_{p}\right)$ and $\sigma_{y}\left(z_{p}\right)$ are shown in Figure 1 and the simulated PSF is depicted in Figure 2. The latter is a $31 \times 31 \times 25$ stack of $\Delta_{x, y}=150[\mathrm{~nm}]$ and $\Delta_{z}=40[\mathrm{~nm}]$. The emission rate of the particle is $2 \cdot 10^{6}$ [photons/sec] and the acquisition time is $1[\mathrm{sec}]$. The readout noise follows a zero-mean Gaussian distribution with a standard deviation of 20 [electron/pixel]. This PSF stack was then represented by B-spline coefficients according to (2).

We generated a sequence of 200 frames at a rate of $100[\mathrm{msec}]$. Each frame consists of a sparse set of particles located at random positions (Figure 3); the total number of particles was 1000 . The size of each frame was $256 \times 256$ pixels. We evaluated our approach using several noise levels; the noise model consists of scatter and readout noise. We also used several $b$-spline kernels with different degree values.

Our localization results are reported in Table 1. The comparison criteria are the RMS (root mean squared) error of the lateral and axial distances between the oracle and the localized particle. The number of oracle particles and the number of localized particles are not necessarily equal. Once we localize a particle, we look for the closest particle in the oracle list and pair them up. The maximum distance between the elements of such a pair cannot be larger than one pixel; this is because we reject localized particles that are too far from their local maxima. Our results indicate a very low RMS value compared to the pixel size, which is in our case $150[\mathrm{~nm}]$. We also report the average value of the single particle localization run time. The first row of Table 1 documents the localization performance for noiseless super-resolution data. It shows that

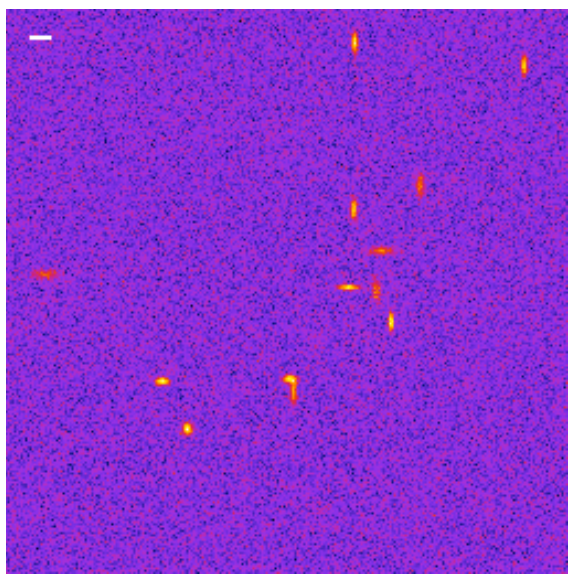

Fig. 3. An example of super resolution data: shown here is a single frame of a sparse set of particles that are located at different depths. Their image is determined by the astigmatic PSF (6). The frame size is $256 \times 256$ and the pixel size is $150[\mathrm{~nm}]$. Noise sources include scatter noise with a mean value that is 10 percent of the emission rate, and read-out noise that has an RMS vale of 20 [electron/pixel]. The mean emission rate is $2 \cdot 10^{6}$ [photon/sec], the acquisition time is $100[\mathrm{msec}]$, and the optical efficiency is 0.033 . The scale bar is 10 pixels wide, corresponding to $1500[\mathrm{~nm}]$.

higher B-spline degree values results in better localization accuracy, as expected from (3). The trade off, however, is computational time due to the larger support of the B-spline kernels. The preferred choice for the $\mathrm{B}$-spline degree is $n=2$; it provides a good balance between accuracy and computational time. The lateral RMS (root mean square) error is less than $10[\mathrm{~nm}]$ for a pixel size of $150[\mathrm{~nm}]$, and less than $25[\mathrm{~nm}]$ in the axial direction. The PSF stack that was used here has a pixel size of $150[\mathrm{~nm}]$ and an axial step size of $40[\mathrm{~nm}]$ (Figure 2). Such performance demonstrates the usefulness of the polynomial B-spline approximation, even if the PSF stack includes noise as is the case for our simulations. We did not include the case of a linear B-spline approximation $(n=1)$ due to the discontinuity of its derivative function at the origin. Note that not all particles have been detected, which is mainly due to configurations where several particles are close to each other, as shown in Figure 3. The average localization time per particle is relatively short - around $30[\mathrm{msec}]$ for B-splines of degree $n=2$. This value can be made even shorter by tuning certain parameters. One can reduce the maximum number of Leveneberg-Marquadt iterations (10 in our work), and relax the stopping condition, which is based on the difference of the fitting error value between two consecutive iterations ( 0.01 for the $\chi^{2}$ function in our work).

Table 1 also reports relatively good localization results for the noisy cases (see last two rows), and the choice of $n=2$ is still the preferred one. As expected, the lateral performance deteriorates as the noise level increases. This is not the case, however, for the axial accuracy, which suggests that the axial $40[\mathrm{~nm}]$ discretization of the PSF is the limiting factor for this quantity. We also observed a decrease in computation time, which is primarily due to the stopping condition 
Table 1. Performance comparison ${ }^{1,2,3}$

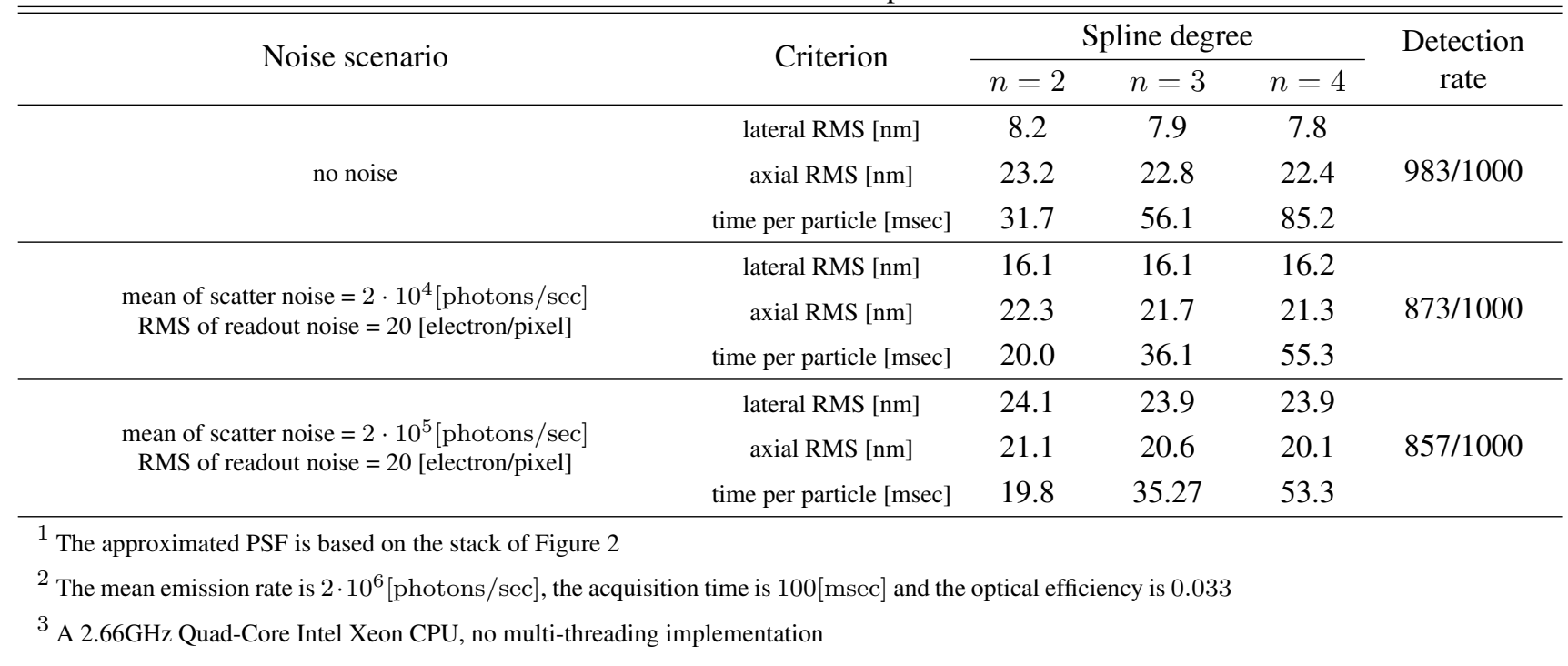

that we used. Due to noise, the Levenberg-Marquardt algorithm quickly reaches a state where a relative improvement of more than 0.01 in the fitting error is no longer possible. The reported run-time values correspond to a sequential implementation. A multi-threaded implementation would decrease the effective localization time substantially, allowing for real-time super-resolution reconstruction. The noise level affects the number of localized particles, too. Some of the localized particles result in SNR values that are too low and some of them are too far away from the local maximum. Such results are discarded from list of detected particles.

\section{CONCLUSIONS}

In this work, we introduced a general and computationally efficient approach to 3-D localization microscopy. The main idea was to create a continuous-domain PSF model using a basis of polynomial B-splines. This representation can be derived from an analytic PSF model or from a measured PSF. The building blocks of our 3-D approximation are compactly supported functions, which allows for a computationally efficient interpolation of the PSF. Our approach can accommodate for any 3-D PSF design, and it does not require an axial calibration curve. It is based on the simple yet effective least-squares criterion. Our results demonstrate the potential of the method for fast and accurate reconstruction of superresolution data.

\section{REFERENCES}

[1] George Patterson, Michael Davidson, Suliana Manley, and Jennifer Lippincott-Schwartz, "Superresolution imaging using single-molecule localization," Annual Review of Physical Chemistry, vol. 61, no. 1, pp. 345-367, 2010.
[2] F. Aguet, D. Van De Ville, and M. Unser, "A maximumlikelihood formalism for sub-resolution axial localization of fluorescent nanoparticles," Optics Express, vol. 13, no. 26, pp. 10503-10522, December 2005.

[3] A. V. Abraham, S. Ram, J. Chao, E. S. Ward, and R .J. Ober, "Quantitative study of single molecule location estimation techniques," Opt. Express, vol. 17, no. 26, pp. 2335223373, December 2009.

[4] S. Wolter, M. Schüttpelz, M. Tscherepanow, S. Van de Linde, M. Heilemann, and M. Sauer, "Real-time computation of subdiffraction-resolution fluorescence images," Journal of Microscopy, vol. 237, no. 1, pp. 12-22, 2010.

[5] R. Henriques, M. Lelek, E. F. Fornasiero, F. Valtorta, C. Zimmer, and M. M. Mhlanga, "QuickPALM: 3D real-time photoactivation nanoscopy image processing in ImageJ," Nature Methods, vol. 7, no. 5, pp. 339-340, May 2010.

[6] Per Niklas Hedde, Jochen Fuchs, Franz Oswald, Jorg Wiedenmann, and Gerd Ulrich Nienhaus, "Online image analysis software for photoactivation localization microscopy," Nat. Meth., vol. 6, no. 10, pp. 689-690, 2009.

[7] B. Huang, W. Wang, M. Bates, and X. Zhuang, "Threedimensional super-resolution imaging by stochastic optical reconstruction microscopy," Science, vol. 319, no. 10, pp. 810813, February 2008.

[8] Ginni Grover, Sean Quirin, Callie Fiedler, and Rafael Piestun, "Photon efficient double-helix psf microscopy with application to 3D photo-activation localization imaging," Biomed. Opt. Express, vol. 2, no. 11, pp. 3010-3020, Nov 2011.

[9] M. Unser, A. Aldroubi, and M. Eden, "B-Spline signal processing: Part II-Efficient design and applications," IEEE Transactions on Signal Processing, vol. 41, no. 2, pp. 834848, February 1993.

[10] B. Zhang, J. Zerubia, and J. C. Olivo-Marin, "Gaussian approximations of fluorescence microscope point-spread function models.," Appl- Opt-, vol. 46, no. 10, pp. 1819-1829, April 2007. 